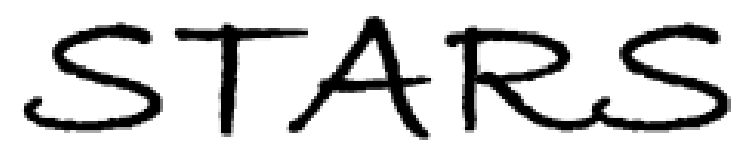

University of Central Florida

STARS

$1-1-2015$

\title{
Passive optical mapping of structural evolution in complex fluids
}

Jose R. Guzman-Sepulveda

University of Central Florida

Kyle M. Douglass

University of Central Florida

Samiul Amin

Neil E. Lewis

Aristide Dogariu

University of Central Florida

Find similar works at: https://stars.library.ucf.edu/facultybib2010

University of Central Florida Libraries http://library.ucf.edu

This Article is brought to you for free and open access by the Faculty Bibliography at STARS. It has been accepted for inclusion in Faculty Bibliography 2010s by an authorized administrator of STARS. For more information, please contact STARS@ucf.edu.

\section{Recommended Citation}

Guzman-Sepulveda, Jose R.; Douglass, Kyle M.; Amin, Samiul; Lewis, Neil E.; and Dogariu, Aristide, "Passive optical mapping of structural evolution in complex fluids" (2015). Faculty Bibliography 2010s. 6557.

https://stars.library.ucf.edu/facultybib2010/6557

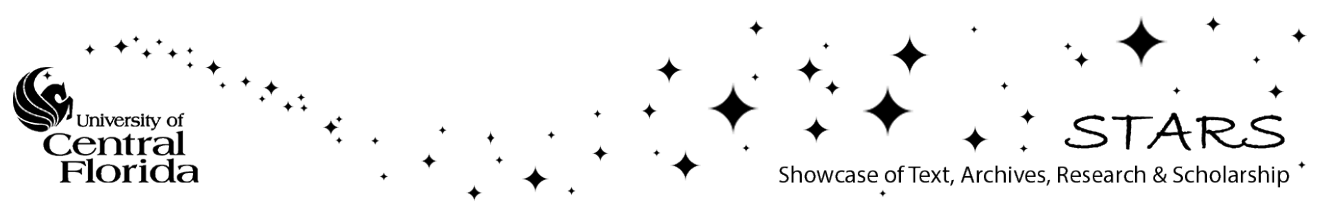




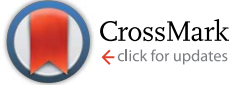

Cite this: RSC Adv., 2015, 5, 5357
Received 1st October 2014

Accepted 2nd December 2014

DOI: $10.1039 / \mathrm{c} 4 \mathrm{ra11627e}$

www.rsc.org/advances

\title{
Passive optical mapping of structural evolution in complex fluids
}

\author{
Jose R. Guzman-Sepulveda, ${ }^{a}$ Kyle M. Douglass, ${ }^{a}$ Samiul Amin, ${ }^{\text {b Neil E. Lewis }}{ }^{\text {b }}$ \\ and Aristide Dogariu*a
}

\begin{abstract}
Self-assembling complex systems exhibit properties that involve a broad spectrum of thermal, structural, morphological, and optical transitions. Various techniques have been used to assess different aspects of the phase transitions in these complex systems. However, because of inherent technical constraints, structural information is usually provided only within narrow ranges of concentrations and temperatures. We show here that by effectively suppressing multiple scattering, low-coherence dynamic light scattering permits assessing the aggregation dynamics of self-assembling systems in a completely passive manner and over ranges of concentration and temperatures well beyond the limits of traditional approaches. The power spectral analysis of scattered intensity fluctuations permits a reliable characterization of multiple relaxation times. We demonstrate that the entire phase diagram can be covered in a consistent way and structural phase transitions can be mapped over a broad optical regime from weak to strong scattering.
\end{abstract}

\section{Introduction}

Self-assembling systems manifest a rich variety of structural and nanoscale morphologies, and rheological, electronic, and optical properties. ${ }^{\mathbf{1 , 2}}$ The large range of mechanical properties which can be designed and engineered makes them suitable for applications such as directing growing in material synthesis ${ }^{3}$ and artificial biological scaffolding in regenerative medicine i.e. tissue engineering. ${ }^{4-6}$ Also, the electronic properties of selfassembling systems play an important role in electronic, photonic, and plasmonic devices for sensing and energy storage. ${ }^{6,7}$ More interestingly, functional materials for sensing and control of biological processes ${ }^{6,8-12}$ as well as therapeutic delivery ${ }^{13-19}$ can also be designed based on the characteristic bio-responsivity of these systems.

Although a variety of protein, nucleic acids (DNA), and peptide systems exist and have been studied in great detail, the polymer-based self-assembling systems, and particularly those conformed by amphiphilic species i.e. block copolymers, are of considerable interest for both the fundamental science and the related technological development and applications. ${ }^{2}$

Self-assembling processes are characterized by critical temperatures and concentrations that define the phase transitions between states with soluble individuals e.g. monomers, and situations where the constituent blocks aggregate forming

${ }^{a}$ CREOL, The College of Optics and Photonics, University of Central Florida, 4304 Scorpius St., Orlando, Florida, 32816, USA. E-mail: adogariu@creol.ucf.edu; Tel: $+1-4078236839$

${ }^{b}$ Malvern Instruments, Columbia, Maryland, USA more complex structures, e.g. micelles. ${ }^{20}$ Following experimentally the structural evolution is rather challenging because physical properties change considerably across such phase transitions. Some of these difficulties, like maintaining the structural integrity during the process, are of general nature. Others depend strictly on the system examined. For instance, the physical property or the contrast mechanism utilized in an experiment, as well as the concentration and the temperature ranges of interest.

The structural transitions in self-assembling systems are being intensively studied using a variety of techniques, among which scattering-based approaches such as small-angle neutron and X-ray scattering (SANS, SAXS), and static and dynamic lightscattering (SLS, DLS) are the most widely used. However, there are inherent experimental constraints, which stem from a variety of thermal, structural, morphological, rheological, and optical transitions experienced by these systems. The situation complicates even more at high concentrations since hydrodynamic interactions are progressively screened as the concentration increases. ${ }^{2}$

Optical scattering-based techniques offer significant experimental flexibility but, so far, suffer from some critical restrictions. Firstly, the samples have to be low-concentrated such that multiple scattering can be avoided. Also, DLS studies normally result in an average decay time which characterizes a dominant relaxation process, ${ }^{21,22}$ this, of course, limits the amount of structural information that can be retrieved. As a result, most common techniques reported so far cover only limited regions of the phase diagrams. 
A standard example of a complex, self-assembling system is the case of aggregating block copolymers. The structural evolution of such complex fluids has been intensely studied by means of SANS, ${ }^{22,23}$ laser temperature jump, ${ }^{24}$ ultrasonic relaxation, ${ }^{25}$ differential scanning calorimetry (DSC), ${ }^{21}$ Fouriertransform infrared spectroscopy (FTIR), ${ }^{21}$ and both SLS and DLS. ${ }^{21,22,25,26}$ The concentration and temperature regimes covered by these techniques are summarized in the schematic phase diagram of Fig. 1 for the specific case of Pluronics L64, a well-studied block copolymer system. ${ }^{27-29}$ As can be seen, it is mainly the low-concentration regime which is accessible to most current experimental procedures. Nevertheless, this situation imposes significant limitations in understanding the role of higher concentrations in, for instance, the evolution of micellar hydrodynamic size and diffusion. ${ }^{\mathbf{2 1 , 2 2}}$

Among the techniques illustrated in Fig. 1, optical approaches are of particular interest because of the flexibility in their practical implementation and potential noninvasiveness. In order to scale to higher concentrations, commonly, colloidal probes are embedded into the system and a global analysis of the dynamic light scattering provides information about the microrheological properties of the complex fluid. ${ }^{26,30}$ This approach has its own limitations when the scattering from the probes starts competing with the intrinsic scattering of the complex system itself. In addition, the presence of the probes alters in certain degree the system's integrity and, complicates the interpretation of the measured dynamics.

In the following we will introduce an optical technique based on low-coherence dynamic light scattering (LC-DLS), which allows monitoring the evolution of the structural changes in complex systems in a completely passive manner. We will show that LC-DLS provides means to effectively isolate the contribution of single scattering events even in the case of media with very high optical densities. ${ }^{31,32}$ Thus, the underlying structural evolution of the system can be mapped over extended ranges of temperature and concentration, which cover a broad optical regime ranging from the weak single-scattering of monomers in aqueous solution to the strong multiple-scattering.

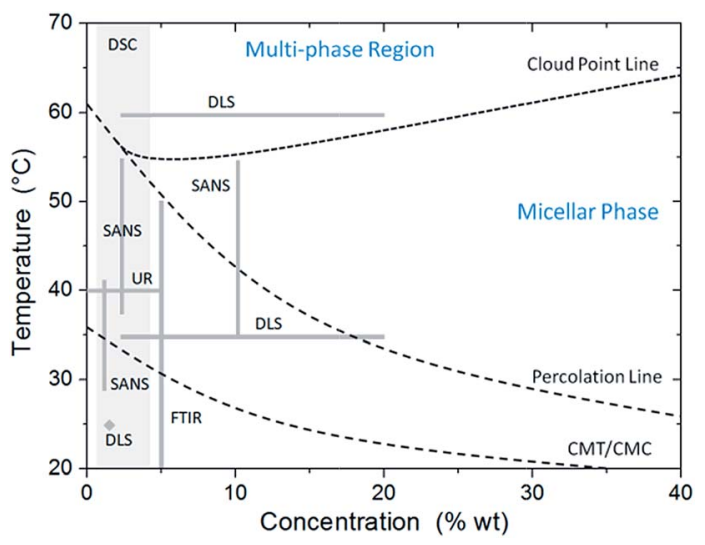

Fig. 1 Schematic of the phase diagram of Pluronics L64, adapted from ref. 27-29. Different experimental techniques cover limited domains of phase diagram as indicated.
The unique capabilities of LC-DLS will be demonstrated on a standard self-assembling system, Pluronics L64. We will show that the structural evolution of this complex fluid can be followed noninvasively, over a broad temperature range, and at concentrations inaccessible to other experimental techniques.

\section{Low-coherence dynamic light scattering}

The fluctuations of the scattered light due to the structural dynamics in the copolymer system were analyzed in the frequency domain by using the LC-DLS technique. ${ }^{33}$

The fiber-based, LC-DLS experimental setup consists of a common path interferometer built around a multimode optical fiber as schematically depicted in Fig. 2. Light from the low coherence source, a super luminescent diode of $\sim 40 \mathrm{~nm}$ bandwidth centered at $670 \mathrm{~nm}$, is launched into a 62.5/125 multimode fiber and then coupled into a 50/50 multimode splitter.

The fiber probe is inserted into the cuvette containing the sample and an analog photodetector measures the intensity of the scattered light coupled back into the fiber. The fluctuations of the electrical signal are digitized using specialized DAQ hardware and further analyzed in the frequency domain.

Due to the inherent coherence gating, this LC-DLS technique allows isolating the back-scattered light from picoliter-sized volumes thus effectively suppressing multiple scattering. This procedure permits isolating the single-scattering component even in optically dense media. ${ }^{31,32}$

The temporal fluctuations of the intensity resulting from the optical mixing between the back-scattered field and the reference field provided by the reflection at the end facet of the fiber probe are analyzed directly in the frequency domain. It has been shown that, in a range of complex fluids, the associated power spectrum of the intensity fluctuations can be represented as a superposition of multiple Lorentzian spectra ${ }^{31,32}$

$$
P(f)=\frac{2}{\pi} \sum_{i=1}^{M} \frac{a_{i} / \tau_{i}}{f^{2}+\left(1 / \tau_{i}\right)^{2}}, \text { subject to } \sum_{i=1}^{M} a_{i}
$$

which is characterized by a distribution of decay times and corresponding amplitudes.

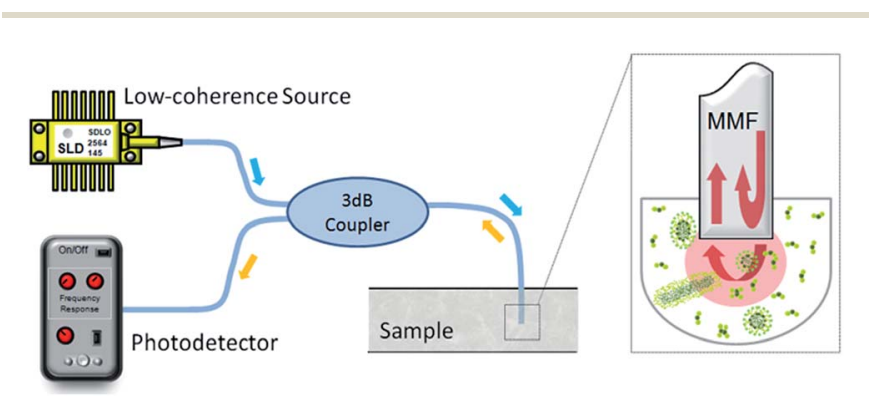

Fig. 2 Fiber optic-based low-coherence dynamic light scattering setup. 


\section{Using LC-DLS to monitor structural changes in block copolymers}

The LC-DLS procedure was tested on a triblock copolymer which constitutes a typical example of self-assembling system. Common material systems are amphiphilic nonionic surfactants, commercially available as Pluronics, which have symmetric structures poly(ethylene oxide)-poly(propylene oxide)-poly(ethylene oxide), PEO-PPO-PEO, or PPO-PEO-PPO.

The self-assembling process in block polymers can be modified not only by changing the intrinsic properties of the polymer but also by changing the solvent properties and the environmental conditions..$^{34,35}$ In addition, the system's evolution is strongly influenced by temperature because, for instance, the micellar structure is directly determined by the temperature-dependent solubility of the conforming blocks. ${ }^{36,37}$ It is also well understood that a transition region exists above the critical micellization temperature where both monomers and micelles can coexist. ${ }^{20,35,38,39}$ Further increase in the temperature leads to more complex interactions such as micelle aggregation, gelation, and even crystalline lattice packing can be produced due to the symmetric nature of the triblock copolymers. ${ }^{2,23,40-47}$ A possible representation of this complicated temperature-dependent process in PEO-PPO-PEO Pluronics systems is schematically illustrated in Fig. 3.

We performed systematic experiments using $\mathrm{PEO}_{13}-\mathrm{PPO}_{30^{-}}$ $\mathrm{PEO}_{13}$, commercial grade Pluronics L64 (BASF). The L64 was used as received, without any additional purification. A multidimensional study was carried out by testing aqueous solutions of Pluronics L64 with concentrations of $10 \% \mathrm{wt}, 20 \% \mathrm{wt}$, and $30 \% \mathrm{wt}$, over an extend temperature range from $25^{\circ} \mathrm{C}$ to $65^{\circ} \mathrm{C}$ in steps of $5{ }^{\circ} \mathrm{C}$. We note that these concentrations are larger than the range accessible to traditional techniques.

Power spectra ranging from $1 \mathrm{~Hz}$ to $50 \mathrm{kHz}$ were recorded over a period of $10 \mathrm{~s}$ of continuous sampling and then a total of 20 such samples were averaged during a total acquisition time of approximately 3 min per temperature point. Fig. 4 illustrates typical LC-DLS experimental power spectra corresponding to scattering from the L64 10\% wt system at different temperatures. According to the phase diagram of L64, these

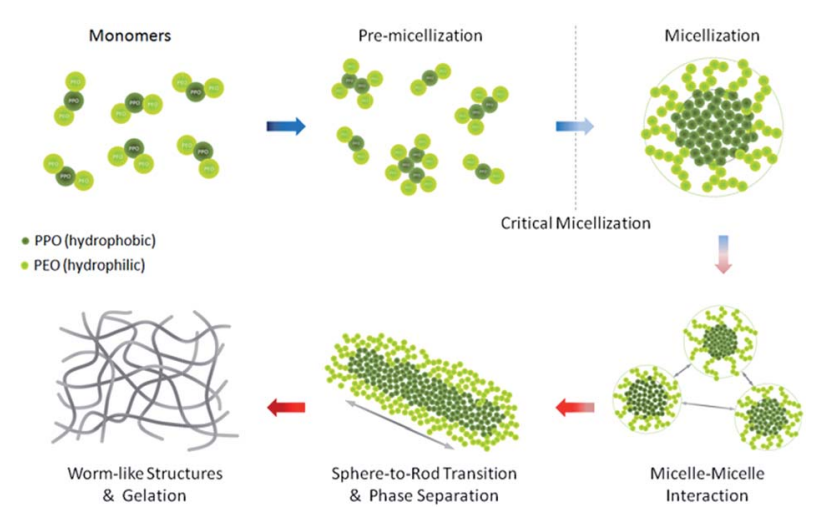

Fig. 3 As temperature increases, the micellization process evolves from a state of dispersed monomers to a gel state.

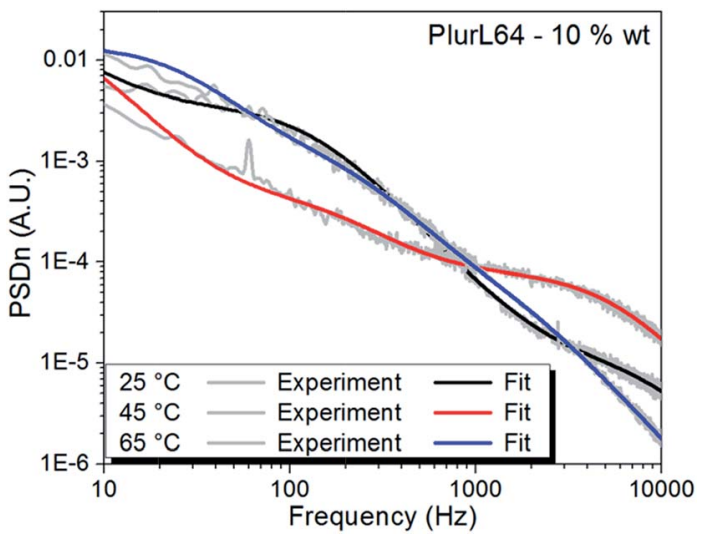

Fig. 4 Typical experimental power spectra and the corresponding multi-Lorentzian fits recorded from Pluronics L64 at 10\% wt concentration and at different temperatures as indicated.

temperatures are close to the critical transition lines: the critical micellization temperature (CMT) at $25{ }^{\circ} \mathrm{C}$, and the so-called "percolation line" and "cloud point" at $45{ }^{\circ} \mathrm{C}$ and $55{ }^{\circ} \mathrm{C}$, respectively. ${ }^{27-29}$

The recorded power spectra were fitted to the multiLorentzian model of eqn (1), as shown in Fig. 4. Even though the fitting algorithm allows weighting many contributions, we found that in most cases the frequency range from $10 \mathrm{~Hz}$ to 10 $\mathrm{kHz}$ could be appropriately described using three Lorentzian components.

As we discussed before, the effective isolation of single scattering regime together with the multiple relaxation times analysis of the power spectrum of intensity fluctuations allowed us to examine the complex polymer system in a consistent manner over a large domain of concentration and temperatures. We emphasize that the entire region of the phase diagram is explored with the same instrument, at the same irradiation power and, most importantly, in a purely passive manner without using external probes.

The collection of relaxation times and amplitudes provided by the multi-Lorentzian analysis is summarized in Fig. 5 . The relative contributions (amplitudes) of the Lorentzian components are normalized according to eqn (1) and are indicated by the color bar. In this representation, the dominant relaxation events can be easily identified and the dynamic evolution of the system can be followed over all accessible time scales.

In this way a comprehensive mapping of the underlying structural evolution can be achieved since the longer relaxation times could relate to the dynamics of large structural components of the complex fluid while the shorter times could potentially describe the fluctuations of smaller species.

Even though the measurement does not provide information about the specific origin of the relaxation events, the collection of times and amplitudes describing the system's dynamics is a direct measurement of the underlying microstructure itself.

Therefore, a number of observations can be made in the context of structural evolution of Pluronics and the features exhibited in the evolution maps can be related to the more 
specific characteristics reported previously for this same complex system.

For instance, at $10 \% \mathrm{wt}$, the temperature range from 30 to 55 ${ }^{\circ} \mathrm{C}$ is dominated by the contribution of the shorter relaxation times while a region where larger features start have a significant contribution can be seen around $60{ }^{\circ} \mathrm{C}$. At $20 \% \mathrm{wt}$, the evolution map shows slight dominance of the short relaxation times from 25 to $35{ }^{\circ} \mathrm{C}$. The activity is dominated by longer relaxation times from $35{ }^{\circ} \mathrm{C}$ to $45{ }^{\circ} \mathrm{C}$ and then the short time relaxation events reappear up to $60{ }^{\circ} \mathrm{C}$. Beyond this point the dynamics is overall described by longer relaxation times. At the highest concentration, the dominant contribution comes from the shorter relaxation times, however, in this case the impact is more evenly distributed over these short time scales and this situation extends up to $60{ }^{\circ} \mathrm{C}$.

The temperature evolution of the spectral components in Fig. 5 offers an inclusive perspective onto the process of structural evolution. For instance, we note that the (color) transition occurring at the lowest temperature relates to the critical
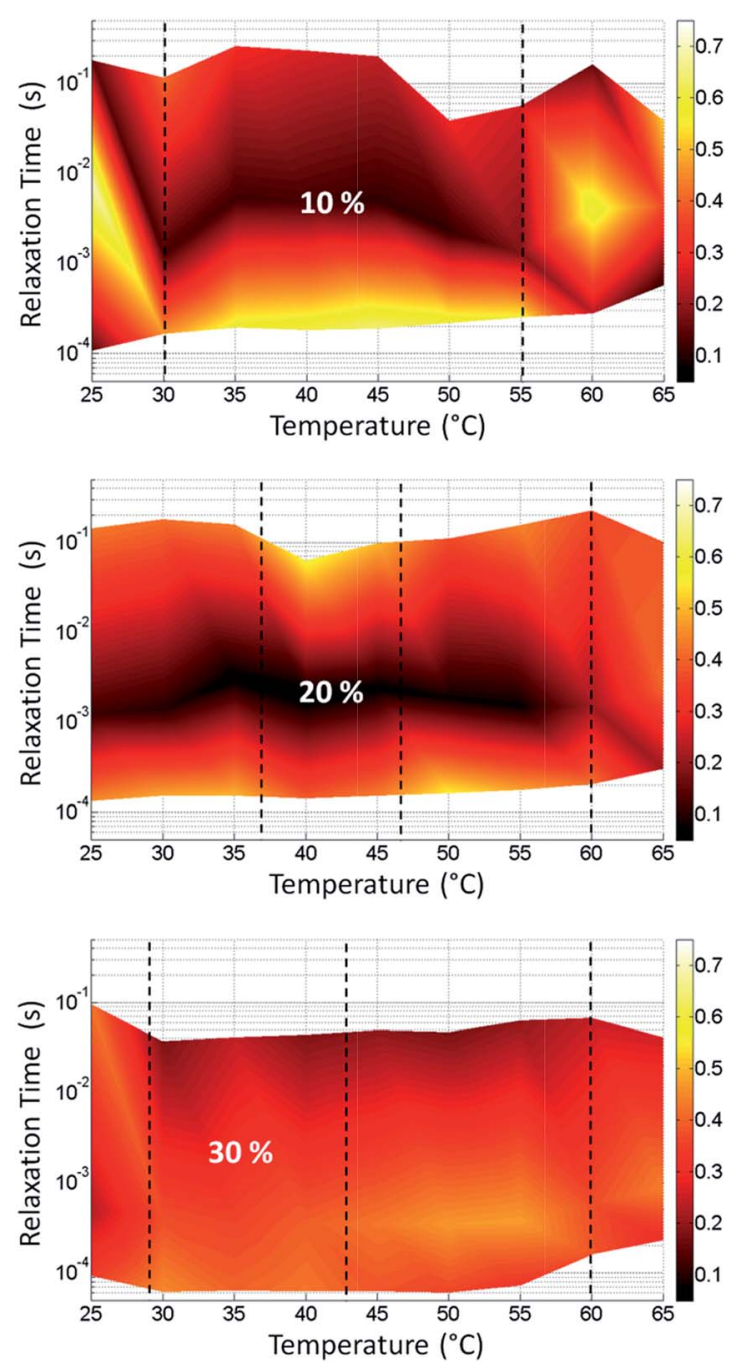

Fig. 5 Temperature evolution of the spectral components determined for Pluronics systems with concentrations as indicated. micellization condition. This transition shifts to lower temperatures as the concentration increases; it shifts from $30-35^{\circ} \mathrm{C}$ for $10 \%$ wt to around $25-30{ }^{\circ} \mathrm{C}$ for $20 \%$ wt and then below $25{ }^{\circ} \mathrm{C}$ for $30 \%$ wt. We note that these temperature ranges found in our experiments are in good agreement with those extrapolated from lower polymer concentrations. ${ }^{21,23,42}$

In the evolution maps shown in Fig. 5, one can also note the transition occurring around $60{ }^{\circ} \mathrm{C}$, which could be indicative of the so-called "cloud point" corresponding to different concentrations. This transition is normally associated to phase separation involving precipitation of the solid compounds but it has also been reported as the signature of the combined effect of anisotropic micellar growth from spherical to rod-like structures, ${ }^{48}$ the onset of strong intermicellar attractive interaction ${ }^{22}$ and clustering, ${ }^{40,49}$ and even the entanglement of worm-like micellar sub-systems. ${ }^{\mathbf{4 2 , 5 0 - 5 2}}$

As mentioned before, the LC-DLS spectra in our experiments are properly described by three spectral components, each one related to a specific relaxation time. The three main relaxation time scales we identified are in good quantitative agreement with other reported data concerning the structural evolution of L64. ${ }^{41,42}$ Our results also confirm that the slow and fast relaxation events are separated approximately by a factor of $10^{3}$ for this particular system. ${ }^{25}$

These spectral components relate to the specific relaxation mechanisms in L64. In general, the fast relaxation mode is associated to the internal changes in the micellar structure independently of concentration. However, the origin of the intermediate and slow relaxation modes depends on the specific conditions. At concentrations higher than 15\%, the attractive interaction between monomers weakens thus inhibiting the process of cluster formation. As a result, the micelles may experience a sphere-to-rod transformation, which may generate entanglement of worm-like structures. ${ }^{22,42,48-52}$

The fast relaxation time found in our experiments occurs around $100 \mu \mathrm{s}$ and is due to the so-called single step equilibrium between micelles and monomers. This relaxation mechanism describes the diffusion of the micelles as an exchange of monomers between the solution and the micelles takes place i.e. monomers join/leave incipient micelles. ${ }^{22-25,40,41}$

The slowest relaxation time is of the order of tenths of seconds and can be associated with the dynamics of large micellar clusters and also possible entanglement of worm-like structures due to the sphere-to-rod transition. ${ }^{22-24,40,49}$ Regardless of its origin, this translates into a low-frequency collective motion.

The intermediate characteristic time scale befalls in the millisecond regime and is the most controversial. Two main types of mechanisms can be associated with this relaxation time: the restoring of the micellar size distribution and the micelle-micelle interactions..$^{23,24,40,49}$ The restoration of the micellar size distribution relates to both the dehydration of the micelle core and the micelle formation/breakdown as the number density of micelles adjusts to the equilibrium distribution. The interactions between micelles include collision, merging, splitting, and dissolving of initially formed micelles. All these mechanisms depend on both the temperature and the 
concentration and manifest in the same time scale regardless of their origin..$^{25,40-42}$

The region of the phase diagram covered by our experiments is significantly extended over a larger domain compared to the other techniques. We emphasize that, for the concentrations considered here (up to $30 \% \mathrm{wt}$ ), the temperature range allows exploring the system dynamics close to the CMT, well within the micellar transition region above the CMT, around the cloud point (CP) where the phase separation starts, and even in a small multiphase region. ${ }^{27-29}$ Due to significant multiple scattering, most of these regions are well beyond the limits of traditional DLS techniques. ${ }^{21,22}$ In fact, with the exception of regions of crystalline packing and lattice formation, LC-DLS allows exploring the entire area of the phase diagram where dynamic fluctuations are present.

We also emphasize that LC-DLS can be used in the assessment of aggregation dynamics in a variety of self-assembling systems and the triblock copolymer studied here is just a standard system that was selected due to the detail information available on its characterization.

\section{Conclusions}

We have shown that LC-DLS allows the passive assessment of the aggregation process in copolymer systems over very large ranges of concentration and temperatures.

Our experiments demonstrate that LC-DLS provides information beyond that typically retrieved from classical dynamic light scattering. We have demonstrated that an extended concentration regime can be covered by an effective suppression of multiple scattering. Moreover, the fiber optic based system used here has a very good signal to noise performance, which allows passive measurements (without inserting external probes) over the full regime of concentrations and temperatures. Thus, the entire domain of the phase diagram can be covered in a consistent way and relevant structural phase transitions can be mapped over a broad optical regime ranging from weak to strong scattering.

The power spectra of the intensity fluctuations have a very good dynamic range, which permits a reliable analysis of multiple relaxation times. Thus, temperature evolution maps of these characteristic times can be created to identify the temporal scale and the progression of predominant scattering components.

Finally, although a measurement based on dynamic light scattering cannot fully discriminate between different mechanisms leading to morphological changes in such complex polymer systems, our experimental approach offers a significantly extended range of structural parameters over which the micellar dynamical activity can be quantified.

\section{Notes and references}

1 W. M. Gelbart and A. Ben-Shaul, J. Phys. Chem., 1996, 100, 13169-13189.

2 T. P. Lodge and M. Muthukumar, J. Phys. Chem., 1996, 100, 13275-13292.
3 B. H. Sohn and B. H. Seo, Chem. Mater., 2001, 13, 1752-1757.

4 K. Rajagopal and J. P. Schneider, Curr. Opin. Struct. Biol., 2004, 14, 480-486.

5 D. N. Woolfson and M. G. Ryadnov, Curr. Opin. Chem. Biol., 2006, 10, 559-567.

6 A. R. Hirst, B. Escuder, J. F. Miravet and D. K. Smith, Angew. Chem., Int. Ed., 2008, 47, 8002-8018.

7 A. Lakshmanan, S. Zhang and C. A. E. Hauser, Trends Biotechnol., 2012, 30, 155-165.

8 O. Ikkala and G. ten Brinke, Science, 2002, 295, 2407-2409.

9 I. Roy and M. N. Gupta, Chem. Biol., 2003, 10, 1161-1171.

10 J. Kopecek, Biomaterials, 2007, 28, 5185-5192.

11 S. Chaterji, I. K. Kwon and K. Park, Prog. Polym. Sci., 2007, 32, 1083-1122.

12 A. Kumar, A. Srivastava, I. Y. Galaev and B. Mattiasson, Prog. Polym. Sci., 2007, 32, 1205-1237.

13 M. C. Branco and J. P. Schneider, Acta Biomater., 2009, 5, 817-831.

14 S. Ganta, H. Devalapally, A. Shahiwala and M. Amiji, J. Controlled Release, 2008, 126, 187-204.

15 S. Kim, J. H. Kim, O. Jeon, I. C. Kwon and K. Park, Eur. J. Pharm. Biopharm., 2009, 71, 420-430.

16 W. Q. Qiu and H. Zhu, Front. Aging Neurosci., 2014, 6, 186.

17 D. Schmaljohann, Adv. Drug Delivery Rev., 2006, 58, 16551670.

18 A. Tschiche, A. Malhotra and R. Haag, Nanomedicine, 2014, 9, 667-693.

19 J. Wang, S. Cui, Y. Bao, J. Xing and W. Hao, Mater. Sci. Eng., C, 2014, 43, 614-621.

20 G. Wanka, H. Hoffmann and W. Ulbricht, Colloid Polym. Sci., 1990, 268, 101-117.

21 J. P. Mata, P. R. Majhi, C. Guo, H. Z. Liu and P. Bahadur, J. Colloid Interface Sci., 2005, 292, 548-556.

22 R. Ganguly, N. Choudhury, V. K. Aswal and P. A. Hassan, J. Phys. Chem. B, 2009, 113, 668-675.

23 L. Yang, P. Alexandridis, D. C. Steytler, M. J. Kositza and J. F. Holzwarth, Langmuir, 2000, 16, 8555-8561.

24 M. J. Kositza, G. D. Rees, A. Holzwarth and J. F. Holzwarth, Langmuir, 2000, 16, 9035-9041.

25 T. Thurn, S. Couderc-Azouani, D. M. Bloor, J. F. Holzwarth and E. Wyn-Jones, Langmuir, 2003, 19, 4363-4370.

26 A. Mustafina, J. Elistratova, L. Zakharova, Y. Kudryashova, O. Bochkova, V. Burilov and S. Soloveva, Colloids Surf., A, 2001, 392, 343-349.

27 P. Alexandridis, Curr. Opin. Colloid Interface Sci., 1996, 1, 490-501.

28 L. Lobry, N. Micali, F. Mallamace, C. Liao and S. H. Chen, Phys. Rev. E: Stat. Phys., Plasmas, Fluids, Relat. Interdiscip. Top., 1999, 60, 7076-7087.

29 X. Zhou, X. Wu, H. Wang, C. Liu and Z. Zhu, Phys. Rev. E: Stat., Nonlinear, Soft Matter Phys., 2011, 83, 041801.

30 C. J. Kloxin and J. H. van Zanten, J. Chem. Phys., 2009, 131, 134904.

31 G. Popescu, A. Dogariu and R. Rajagopalan, Phys. Rev. E: Stat., Nonlinear, Soft Matter Phys., 2002, 65, 041504.

32 I. Sohn, R. Rajagopalan and A. Dogariu, J. Colloid Interface Sci., 2004, 269, 503-513. 
33 G. Popescu and A. Dogariu, Opt. Lett., 2001, 26, 551-553. 34 P. Linse, Macromolecules, 1993, 26, 4437-4449.

35 P. Alexandridis, J. F. Holzwarth and T. A. Hatton, Macromolecules, 1994, 27, 2414-2425.

36 J. S. Higgins and H. Benoît, Polymers and neutron scattering, Clarendon Press, Oxford, 1994, p. 456.

37 L. Jia, C. Guo, L. Yang, J. Xiang, Y. Tang, C. Liu and H. Liu, J. Colloid Interface Sci., 2010, 345, 332-337.

38 Z. Zhou and B. Chu, J. Colloid Interface Sci., 1988, 126, 171180.

39 P. Alexandridis and J. F. Holzwarth, Langmuir, 1997, 13, 6074-6082.

40 I. Goldmints, J. F. Holzwarth, K. A. Smith and T. A. Hatton, Langmuir, 1997, 13, 6130-6134.

41 M. J. Kositza, C. Bohne, P. Alexandridis, T. A. Hatton and J. F. Holzwarth, Macromolecules, 1999, 32, 5539-5551.

42 M. J. Kositza, C. Bohne, P. Alexandridis, T. A. Hatton and J. F. Holzwarth, Langmuir, 1999, 15, 322-325.
43 E. Hecht and H. Hoffmann, Langmuir, 1994, 10, 86-91.

44 E. Hecht and H. Hoffmann, Colloids Surf., A, 1995, 96, 181197.

45 E. Hecht, K. Mortensen, M. Gradzielski and H. Hoffmann, J. Phys. Chem., 1995, 99, 4866-4874.

46 I. Paterson, J. Armstrong, B. Chowdhry and S. Leharne, Langmuir, 1997, 13, 2219-2226.

47 P. Alexandridis, T. Nivaggioli and T. A. Hatton, Langmuir, 1995, 11, 1468-1476.

48 V. Castelletto, P. Parras, I. W. Hamley, P. Bäverbäck, J. S. Pedersen and P. Panine, Langmuir, 2007, 23, 6896-6902.

49 D. Bedrov, C. Ayyagari and G. D. Smith, J. Chem. Theory Comput., 2006, 2, 598-606.

50 M. Almgren, P. Bahadur, M. Jansson, P. Li, W. Brown and A. Bahadur, J. Colloid Interface Sci., 1992, 151, 157-165.

51 P. Bahadur and K. Pandya, Langmuir, 1992, 8, 2666-2670.

52 Z. Zhou and B. Chu, Macromolecules, 1994, 27, 2025-2033. 